\title{
Spatiotemporal distribution of caudal-type homeobox proteins during development of the hindgut and anorectum in human embryo
}

Xiao Bing Tang, Tao Zhang, Wei Lin Wang, Zheng Wei Yuan, Yu Zuo Bai

Background: The objectives of this study were to determine the spatiotemporal distribution of human caudal-type homeobox proteins CDX1, CDX2 and CDX4 during development of the hindgut and anorectum in the embryo and to explore the possible roles of CDX genes during morphogenesis of the hindgut and anorectum.

Methods:Embryos(89) were cut into sections serially and sagittally. From gestation weeks $4-9$, CDX1, CDX2 and CDX4 proteins were detected on the caudal midline by immunohistochemical staining.

Results: During week4, extensive immunoreactivity of CDX1, CDX2 and CDX4 was detected in the dorsal urorectal septum, urogenital sinus and hindgut. From weeks 5-7, CDX1-, CDX2- and CDX4- positive cells were detected mainly in the mesenchyme of the urorectal septum and hindgut. The levels of CDX2 and CDX4 immunoreactivity were lower compared to CDX1. During weeks 8 and 9, the anorectal epithelium stained positive for CDX1 and CDX4, and the anal epithelium was positive for CDX2.

Conclusions: The CDX proteins are constantly distributed during development of the hindgut and anorectum and exhibit overlapping distribution patterns in the cloaca/hindgut, suggesting they are important in the morphogenesis of the human hindgut and anorectum. CDX genes might be involved in development of the anorectal epithelium after the rectum has separated from the urorectal septum. 


\section{Spatiotemporal distribution of caudal-type homeobox proteins during}

\section{2 development of the hindgut and anorectum in human embryo}

4 Xiao Bing Tang ${ }^{\mathrm{a}}$, Tao Zhang ${ }^{\mathrm{b}}$, Wei Lin Wang ${ }^{\mathrm{a}}$, Zheng Wei Yuan ${ }^{\mathrm{c}}$, Yu Zuo Bai ${ }^{\mathrm{a}}$

5

$6 \quad{ }^{a}$ Department of Pediatric Surgery, Shengjing Hospital, China Medical University, Shenyang

7 110004, P.R. China

8 bepartment of General Surgery, Affiliated Hospital of Hebei University, Baoding 071000, P.R.

9 China

$10{ }^{\mathrm{c}}$ The Key Laboratory of Health Ministry for Congenital Malformation, Shenyang 110004, P.R.

11 China

12

13 Correspondence to: Professor Yu Zuo Bai, Department of Pediatric Surgery, Shengjing Hospital,

14 China Medical University, 36 Sanhao Street, Heping District, Shenyang, P.R. China 110004.

15 Tel.: 0086-24-9661557111; fax: 0086-24-23892617

16 E-mail: baiyz@sj-hospital.org 
17 Abstract

18 Background: The objectives of this study were to determine the spatiotemporal distribution of human caudal-

19 type homeobox proteins CDX1, CDX2 and CDX4 during development of the hindgut and anorectum in the

20 embryo and to explore the possible roles of CDX genes during morphogenesis of the hindgut and anorectum.

21 Methods: Embryos (89) were cut into sections serially and sagittally. From gestation weeks 4 - 9, CDX1,

22 CDX2 and CDX4 proteins were detected on the caudal midline by immunohistochemical staining.

23 Results: During week 4, extensive immunoreactivity of CDX1, CDX2 and CDX4 was detected in the dorsal

24 urorectal septum, urogenital sinus and hindgut. From weeks 5-7, CDX1-, CDX2- and CDX4-positive cells

25 were detected mainly in the mesenchyme of the urorectal septum and hindgut. The levels of CDX2 and

26 CDX4 immunoreactivity were lower compared to CDX1. During weeks 8 and 9 , the anorectal epithelium

27 stained positive for CDX1 and CDX4, and the anal epithelium was positive for CDX2.

28 Conclusions: The CDX proteins are constantly distributed during development of the hindgut and anorectum

29 and exhibit overlapping distribution patterns in the cloaca/hindgut, suggesting they are important in the

30 morphogenesis of the human hindgut and anorectum. CDX genes might be involved in development of

31 the anorectal epithelium after the rectum has separated from the urorectal septum. 
34 Introduction

35 Anorectal malformations (ARMs) are among the most common human congenital anomalies,

36 occurring in approximately 1/5000 -1/1500 live births (Van der Putte 1986), with adverse

37 influences on patient quality of life (Peña et al 1998; Bai et al 2000; Levitt et al 2005). ARMs are

38 complex diseases and their etiology, embryology and pathogenesis remain controversial and

39 poorly understood (Wang et al 2015). ARMs might result from mutations in a variety of genes

40 and the expression patterns of several genes during various stages of gastrulation have helped to

41 clarify the molecular basis of this condition (van de Ven et al 2011; Warot et al 1997; Ramalho-Santos et

42 al 2000; Kimmel et al 2000; Seifert et al 2008; Garcia-Barceló et al 2008; Wang et al 2009; Dravis C et al

43 2004.).

44 Caudal-type homeobox ( $C d x)$ genes show highly restricted expression patterns at the onset of

45 gastrulation, suggesting their involvement in the formation of the digestive tract (McGinnis and

46 Krumlauf 1992; Silberg et al 2000; Bonner et al 1995). Earlier studies on the spatiotemporal

47 expression patterns of $C d x 1, C d x 2$ and $C d x 4$ in rat embryo suggested downregulation of these

48 genes during separation of the cloaca into the rectum and urethra was related to ARM

49 development (Zhang et al 2009; Tang et al 2014a, Tang et al 2014b). Cdx2-/- mice displayed severe

50 hindgut abnormalities with failure of colon development and complete terminal blockage (Gao et al 2009), and

$51 \mathrm{Cd} 2+/-; \mathrm{Cd} \times 4-/-$ mice manifested cloacal septation and anorectal defects, including imperforate

52 anus (van de Ven et al 2011). Together these results suggest $C d x$ genes are related to anorectal

53 morphogenesis in animal models. Distribution patterns of the equivalent human CDX proteins,

54 however, have not been investigated in relation to embryogenesis of the cloaca, hindgut and

55 anorectum, and the involvement of these genes in human cloacal development and their effects 
56 on human embryonic hindgut/anorectal development are unknown. This study was designed to

57 determine the distribution patterns of human CDX proteins and their possible roles in

58 hindgut/anorectal morphogenesis. We conducted a systematic study of the spatiotemporal

59 localization of human CDX proteins in normal embryos, with special emphasis on embryonic

60 stages from development weeks 4 -9, which represent the crucial time points in human

61 hindgut/anorectal development.

62

\section{Materials and Methods}

64 Sample preparation

65 The study protocol was in accordance with the World Medical Association Declaration of

66 Helsinki and was approved by the China Medical University Ethics Committee (no. 200(7)

67 PS14). A total of 89 phenotypically normal human embryos of 4 - 9 weeks gestation were

68 obtained, with written informed consent, from 22 -35 years old women with no history of

69 hereditary disease who were undergoing elective chemically induced/atraumatic curettage

70 termination of unplanned pregnancy (Table 1). Embryos were washed immediately in cold

71 phosphate sodium-buffered saline (PBS pH 7.4) and then fixed in 4\% PBS buffered

72 paraformaldehyde $(\mathrm{pH} 7.4)$ at $4^{\circ} \mathrm{C}$ for $24 \mathrm{~h}$. Samples were dehydrated, embedded in paraffin and

73 then cut sagittally into $4-\mu \mathrm{m}$ thick sections.

74 Immunohistochemical staining 
75 Endogenous peroxidase activity was blocked by incubation in 3\% $\mathrm{H} 2 \mathrm{O} 2$ at room temperature for $20 \mathrm{~min}$.

76 Antigens were retrieved by heating the slides in $10 \mathrm{mmol} / \mathrm{L}$ sodium citrate buffer $\left(\mathrm{pH} 6.0\right.$ ) at $98^{\circ} \mathrm{C}$ for $10 \mathrm{~min}$.

77 Sections were treated and incubated with primary rabbit polyclonal anti-CDX1 antibody (LSBio/LS-

$78 \mathrm{C} 180091 / 48877 ; 1: 200)$, primary mouse monoclonal anti-CDX2 antibody (LSBio/LS-

79 B4299/38994; 1:50) or primary rabbit polyclonal anti-CDX4 antibody (LSBio/LS-C30413/51929;

80 1:200) and horseradish peroxidase-conjugated secondary antibody (Santa Cruz Biotechnology Inc., Calif.,

81 USA). Antibodies were incubated in PBS, supplemented with $10 \%$ goat serum. Sections were incubated with

82 primary antibodies at $4^{\circ} \mathrm{C}$ for $16 \mathrm{~h}$ and then incubated with secondary antibody for $20 \mathrm{~min}$ at room temperature.

83 Immunoreactions were visualized using 3,3P-diaminobenzidine (Sigma, Manchester, UK) as a chromogen.

84 Sections were counterstained with hematoxylin and reviewed independently by two pathologists; the results

85 were agreed by consensus. Negative controls were performed by either omitting the primary or secondary

86 antibody.

87 The overall intensity of the immunostaining reaction was evaluated and categorized as "_"

88 to “+”:-negative staining (no colored stain); \pm , weak positive staining (light-yellow stain); +, positive staining

89 (yellow-brown stain).

90

91 Results

92 In embryos at gestation week 4, a triangular early cloaca was observed at the anterior aspect of the caudal

93 end of the spine. Immunoreactivity specific to CDX1, CDX2 or CDX4 was detected mainly in the dorsal

94 urorectal septum (URS), urogenital sinus (UGS) and hindgut. The ventral URS and the cloacal membrane (CM)

95 were negative for CDX1, CDX2 and CDX4 (Figures 1).

96 During week 5, the cloaca was divided into the UGS ventrally and the hindgut dorsally. CDX1-, CDX2- and 
97 CDX4-positive cells were detected mainly in the mesenchyme of the URS and hindgut. CM and UGS were

98 negative for CDX1, CDX2 and CDX4. CDX2 and CDX4 immunoreactivity levels were lower compared to

99 CDX1 (Figures 2).

100 During gestation weeks 6 and 7, the CM was very thin and elongated. The immunoreactivity levels and

101 distributions of CDX proteins were similar to those seen during week 5 (Figures 3-4).

102 During gestation weeks 8 and 9, the rectum became separated completely from the UGS and CDX1-,

103 CDX2- and CDX4-positive cells disappeared from the mesenchyme. At the same time, the anorectal

104 epithelium was positive for CDX1 and CDX4, and CDX1 immunoreactivity decreased gradually from the

105 proximal rectum to the anus. The anal epithelium was positive for CDX2 (Figures 5-6).

106 The distribution patterns of CDX protein are given in Table 2.

107

108 Discussion

109 This study showed human CDX1, CDX2 and CDX4 proteins were distributed from gestation weeks $4-9$ in

110 a spatiotemporal pattern during embryonic anorectal morphogenesis. During gestation week 4, CDX1, CDX2

111 and CDX4 were detected in the dorsal URS, UGS and hindgut. From weeks 5 - 7, they were distributed mainly

112 in the mesenchyme of the URS and hindgut. After the anorectum and the UGS opened to the amniotic cavity

113 during week 8 , the distribution of $\mathrm{CDX} 1, \mathrm{CDX} 2$ and $\mathrm{CDX} 4$ in the mesenchyme decreased, and they were

114 detected in the epithelium of the anorectum/anus. Furthermore, CDX1, CDX2 and CDX4 showed spatially

115 specific distribution patterns in human embryos. They distributed prominently in the dorsal parts of the cloaca

116 that developed into the anorectum, but distributed weakly or almost absent from the ventral part of the cloaca,

117 which develops into the UGS. These results suggest CDX genes might contribute to the development of the

118 cloaca/hindgut and anorectum in the human embryo. 
119 The cloaca is a key feature in the normal morphogenesis of the anorectum (Zhang et al 2011).

120 Despite their likely complex multifactorial etiology, maldevelopment of the URS and CM is generally thought

121 to be responsible for ARMs (Bai et al 2004, Qi et al 2002). The results of this study suggested CDX1,

122 CDX2 and CDX4 were active in the URS during separation of the cloaca from gestation weeks $4-$

123 7, but their distribution level decreased after the anorectum and UGS opened to the amniotic cavity in week 8.

124 These findings provide further evidence for the involvement of CDX genes in the maintenance and pattern

125 formation of the URS during development of the hindgut and anorectum. Abnormal expression of CDX

126 genes might impair development of the URS and subsequent morphogenesis of the

127 cloaca/hindgut and could be involved in the development of human ARMs.

$128 \mathrm{Cdx} 1, \mathrm{Cdx} 2$ and $\mathrm{Cdx} 4$ exhibited overlapping expression patterns in the posterior embryo in animal models,

129 and had related functions with regard to their roles in pattern formation of the paraxial mesoderm (Beck 2004;

130 Lohnes 2003; Savory et al 2009; van den Akker et al 2002; van Nes et al 2006). CDX proteins also exhibit

131 highly overlapping distribution patterns during human cloaca/hindgut development (Figure 7); CDX1,

132 CDX2 and CDX4 distributed in the same part of the human cloaca from gestation weeks 4-7, indicating CDX

133 genes might have cooperative functions during development of the human hindgut and anorectum. Cross-

134 regulatory interactions might exist among $C d x$ genes with regard to anorectal development. van

135 de Ven (van de Ven et al 2011) showed Cdx1-/-and Cdx4-/- mice did not develop anorectal defects,

136 whereas $\mathrm{Cd} \times 2-/-$ mice did, suggesting $\mathrm{Cd} \times 2$ has a more prominent morphogenetic role in mice compared to

$137 \mathrm{Cdx} 1$ or $\mathrm{Cdx} 4$. However, immunoreactivity of CDX1 protein was stronger compared to CDX2 and CDX4 in

138 the cloaca/hindgut and anorectum in this study, suggesting CDX1 might have a more prominent morphogenetic

139 role in the human anorectum compared to CDX2 and CDX4.

$140 C D X$ genes might be involved in development of the anorectal epithelium. We showed CDX 
141 proteins distributed in the anorectal/anal epithelium during gestation weeks 8 and 9. $C d x 1$ and $C d x 2$

142 exhibited transcriptional specificity in the intestine (Grainger et al 2013), and $C d x 2$ has been

143 shown to be crucial for the expression of signaling molecules, epithelial-mesenchymal

144 interactions and intestinal proliferation patterns (Gao et al 2009; Grainger et al 2010). Cdx4 is a

$145 C d x 2$ target gene (Savory et al 2011). The results of this study and earlier work indicate CDX genes

146 might have a role in development of the anorectal epithelium after the rectum has separated from

147 the UGS.

148 The distribution patterns of CDX proteins in humans differ markedly from those of the equivalent proteins in

149 animal models (Figure 7). Cdx1, Cdx2, and Cdx4 are expressed in the developing hindgut endoderm of mice,

150 whereas only Cdx1 and Cdx2 are expressed up to the late gestation and postnatal stages (Beck 2002). Our

151 earlier studies on the spatiotemporal localization patterns of $\mathrm{Cdx} 1, \mathrm{Cdx} 2$ and $\mathrm{Cdx} 4$ proteins in rat

152 embryo suggested Cdx1-, Cdx2- and Cdx4-positive cells were located in the cloacal/hindgut epithelium

153 during development of the hindgut and anorectum (Zhang et al 2009, Tang et al 2014a, Tang et al

154 2014b) (Figure 7). In animal models, Cdx proteins are expressed in the epithelium of the cloaca/hindgut,

155 whereas in human embryo, CDX1, CDX2 and CDX4 proteins located mainly in the peri-cloacal mesenchyme

156 (PCM) during anorectal development (gestation weeks 4-7). Asymmetric growth and patterning of the

157 cloacal mesoderm results in division of the cloacal cavity and formation of a genital tubercle (Wang C et al.

158 2011). The cloaca is a key feature in the normal morphogenesis of human anorectum (Zhang et al 2011).

159 Cloacal membrane (CM) and urorectal septum (URS) play a crucial role on the cloacal embryogenesis (Zhang

160 et al 2011). These results indicated CDX genes might have a role in dorsoventral patterning of the PCM and

161 suggest misexpression of CDX genes might contribute to maldevelopment of the PCM and

162 subsequent impairment of human hindgut and anorectum morphogenesis. 


\section{Conclusions}

165 In conclusion, the results of this study demonstrate CDX proteins distributed throughout the crucial

166 period of hindgut and anorectum development in the human embryo. These proteins exhibit overlapping

167 distribution patterns in the cloaca/hindgut, suggesting they could have a pivotal role in the morphogenesis of

168 the cloaca, hindgut and anorectum, and might be involved in development of the anorectal epithelium.

169 Further studies are required to investigate the role of human CDX genes in anorectal development and their

170 potential involvement in ARMs.

171

172

173

\section{References}

175 Bai YZ, Chen H, Yuan ZW, Wang W. 2004. Normal and abnormal embryonic development of the anorectum in 176 rats. J Pediatr Surg 39:587-590.

177 Bai Y, Yuan Z, Wang W, Zhao Y, Wang H, Wang W. 2000. Quality of life for children with fecal incontinence

178 after surgically corrected anorectal malformation. J Pediatr Surg 35:462-464.

179 Beck F. 2002. Homeobox genes in gut development. Gut 51:450-454.

180 Beck F. 2004. The role of Cdx genes in the mammalian gut. Gut 53:1394-1396.

181 Bonner CA, Loftus SK, Wasmuth JJ. 1995. Isolation, characterization, and precise physical localization of 182 human CDX1, a caudal-type homeobox gene. Genomics 28:206-211. 
183 Dravis C, Yokoyama N, Chumley MJ. 2004. Bidirectional signaling mediated by ephrin-B2 and

184 EphB2 controls urorectal development. Dev Biol 271: 272-290.

185 Garcia-Barceló MM, Chi-Hang Lui V, Tam P. 2008. Mutational analysis of SHH and GLI3 in

186 anorectal malformations. Birth Defects Res A Clin Mol Teratol 82:644-648.

187 Gao N, White P, Kaestner KH. 2009. Establishment of intestinal identity and epithelial-mesenchymal signaling

188 by Cdx2. Dev Cell 16:588-599.

189 Grainger S, Hryniuk A, Lohnes D. 2013. Cdx1 and Cdx2 Exhibit Transcriptional Specificity in the Intestine.

$190 \quad$ PLoS One 8:e54757.

191 Grainger S, Savory JG, Lohnes D. 2010. Cdx2 regulates patterning of the intestinal epithelium. Dev Biol

$192 \quad 339: 155-165$.

193 Kimmel SG, Mo R, Hui CC. 2000. New mouse models of congenital anorectal malformations. J

194 Pediatr Surg 35: 227-230.

195 Levitt MA, Peña A. 2005. Outcomes from the correction of anorectal malformations. Curr Opin Pediatr 17:394-

196401.

197 Lohnes D. 2003. The Cdx1 homeodomain protein: an integrator of posterior signaling in the mouse. Bioessays

$198 \quad 25: 971-980$

199 McGinnis W, Krumlauf R. 1992. Homeobox genes and axial patterning. Cell 68:283-302.

200 Peña A, Guardino K, Tovilla JM, Levitt MA, Rodriguez G, Torres R. 1998. Bowel management for fecal

201 incontinence in patients with anorectal malformations. J Pediatr Surg 33:133-137.

202 Qi BQ, Beasley SW, Frizelle FA. 2002. Clarification of the processes that lead to anorectal malformations in

203 the ETU-induced rat model of imperforate anus. J Pediatr Surg 37:1305-1312.

204 Ramalho-Santos M, Melton DA, McMahon AP. 2000. Hedgehog signals regulate multiple aspects

205 of gastrointestinal development. Development 127: 2763-2772. 


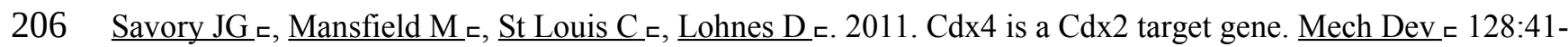
20748.

208 Savory JG, Pilon N, Grainger S, Sylvestre JR, Beland M, Houle M, Oh K, Lohnes D. 2009. Cdx1 and Cdx2 are 209 functionally equivalent in vertebral patterning. Dev Biol 330:114-122.

210 Seifert AW, Harfe BD, Cohn MJ. 2008. Cell lineage analysis demonstrates an endodermal origin

211 of the distal urethra and perineum. Dev Biol 318:143-152.

212 Silberg DG, Swain GP, Suh ER, Traber PG. 2000. Cdx1 and Cdx2 expression during intestinal development.

213 Gastroenterology 119: 961-971.

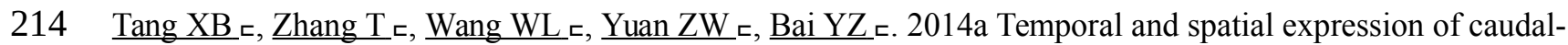

215 type homeobox gene-2 during hindgut development in rat embryos with ethylenethiourea-induced anorectal

216 malformations. Cell Tissue Res $\simeq$ 357:83-90.

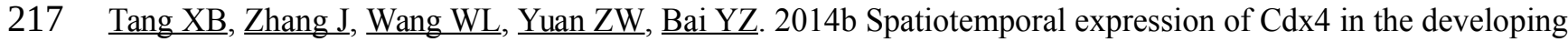

218 anorectum of rat embryos with ethylenethiourea-induced anorectal malformations. Cells Tissues

219 Organs 199:212-220.

220 van de Ven C, Bialecka M, Neijts R, Young T, Rowland JE, Stringer EJ, Van Rooijen C, Meijlink F, Nóvoa A,

221 Freund JN, Mallo M, Beck F, Deschamps J. 2011. Concerted involvement of Cdx/Hox genes and Wnt

222 signaling in morphogenesis of the caudal neural tube and cloacal derivatives from the posterior growth

223 zone. Development 138:3451-3462.

224 van den Akker E, Forlani S, Chawengsaksophak K, de Graaff W, Beck F, Meyer BI, Deschamps J. 2002. Cdx1

225 and $\mathrm{Cdx} 2$ have overlapping functions in anteroposterior patterning and posterior axis elongation.

226 Development 129:2181-2193.

227 Van der Putte SC. 1986. Normal and abnormal development of the anorectum. J Pediatr Surg 21:434-440. 
229 development and reveals an essential role of $\mathrm{Cdx}$ genes in the ontogenesis of the placental labyrinth in mice.

$230 \quad$ Development 133:419-428.

231 Wang C, Gargollo P, Guo C, Tang T, Mingin G, Sun Y, Li X. 2011. Six1 and Eya1 are critical regulators of peri-

232 cloacal mesenchymal progenitors during genitourinary tract development. Dev Biol 360:186-194.

233 Wang C, Li L, Cheng W. 2015. Anorectal malformation: the etiological factors. Pediatr Surg Int $234 \quad 31: 795-804$.

235 Wang DJ, Bai YZ, Wang WL. 2009. Expression of EphB2 in the development of anorectal

236 malformations in fetal rats. J Pediatr Surg 44:592-599.

237 Warot X, Fromental-Ramain C, Fraulob V, Chambon P, Dollé P. 1997. Gene dosage-dependent

238 effects of the Hoxa-13 and Hoxd-13 mutations on morphogenesis of the terminal parts of the

239 digestive and urogenital tracts. Development 124: 4781-4791.

240 Zhang T, Bai YZ, Zhang D, Zhang SW, Wang DJ, Jia HM, Yuan ZW, Wang WL. 2009. Temporal and spatial

241 expression of caudal-type homeobox gene-1 in the development of anorectal malformations in rat embryos.

$242 \quad$ J Pediatr Surg 44:1568-1574.

243 Zhang T, Zhang HL, Wang DJ, Tang XB, Jia HM, Bai YZ, Yuan ZW, Wang WL. 2011. Normal development of

244 hindgut and anorectum in human embryo. Int J Colorectal Dis 26:109-116.

246 Table 1 Distribution of embryos at different ages

\begin{tabular}{cccccccc}
\hline Gestational age (weeks) & 4 & 5 & 6 & 7 & 8 & 9 & Total \\
\hline Number of embryos & 9 & 15 & 16 & 18 & 16 & 15 & 89 \\
\hline
\end{tabular}




\section{Gestational age}

Protein

\begin{tabular}{|c|c|c|c|}
\hline (weeks) & CDX1 & $\mathrm{CDX} 2$ & $\mathrm{CDX} 4$ \\
\hline \multicolumn{4}{|l|}{ Hindgut } \\
\hline 4 & + & + & + \\
\hline 5-7 (epithelium) & - & - & - \\
\hline 5-7 (mesenchyme) & + & \pm & \pm \\
\hline 8-9 (epithelium) & Anorectum + & Anus + & Anorectum+ \\
\hline 8-9 (mesenchyme) & - & - & - \\
\hline \multicolumn{4}{|l|}{ URS } \\
\hline 4 & dURS + & $\mathrm{dURS} \pm$ & $\mathrm{dURS} \pm$ \\
\hline 5-7 (epithelium) & - & - & - \\
\hline 5-7 (mesenchyme) & + & \pm & \pm \\
\hline 8-9 (epithelium) & - & - & - \\
\hline 8-9 (mesenchyme) & - & - & - \\
\hline \multicolumn{4}{|l|}{ UGS } \\
\hline 4 & + & \pm & \pm \\
\hline 5-7 (epithelium) & - & - & - \\
\hline 5-7 (mesenchyme) & - & - & - \\
\hline 8-9 (epithelium) & - & - & - \\
\hline 8-9 (mesenchyme) & - & - & - \\
\hline
\end{tabular}

$249+$, Positive staining; \pm , weak positive staining; - , negative staining; dURS, dorsal URS.

250

251 Figure 1. Spatiotemporal distribution of caudal-type homeobox proteins during gestation

252 week 4.

253

254

255

256 During gestation week 4, immunoreactivity specific to CDX1, CDX2 or CDX4 was detected mainly in the

257 dorsal urorectal septum (URS), urogenital sinus (UGS) and hindgut (H). The ventral URS and the cloacal

258 membrane (CM) were negative for CDX1, CDX2 and CDX4.

259 (URS urorectal septum, $\boldsymbol{U} \boldsymbol{G S}$ urogenital sinus, $\boldsymbol{C M}$ cloacal membrane, $\boldsymbol{H}$ hindgut). 
260 Red rectangles in $\mathbf{a}, \mathbf{d}, \mathbf{g}$ and $\mathbf{j}$ are shown at higher magnification in $\mathbf{b}, \mathbf{e}, \mathbf{h}$ and $\mathbf{k}$, respectively. Green

261 rectangles in $\mathbf{b}, \mathbf{e}, \mathbf{h}$ and $\mathbf{k}$ are shown at higher magnification in $\mathbf{c}, \mathbf{f}, \mathbf{i}$ and $\mathbf{l}$, respectively.

262 Original magnification $100 \times(\mathbf{a}, \mathbf{d}, \mathbf{g}$ and $\mathbf{j}), 200 \times(\mathbf{b}, \mathbf{e}, \mathbf{h}$ and $\mathbf{k})$ and $400 \times(\mathbf{c}, \mathbf{f}, \mathbf{i}$ and $\mathbf{l})$.

263

264 Figure 2. Spatiotemporal distribution of caudal-type homeobox proteins during gestation

265 week 5.

266

267

268

269

During gestation week 5, CDX1-, CDX2- and CDX4-positive cells were detected mainly in the mesenchyme

of the urorectal septum (URS) and hindgut (H). The cloacal membrane (CM) and urogenital sinus (UGS) were

negative for CDX1, CDX2 and CDX4. CDX2 and CDX4 immunoreactivity levels were lower compared to

CDX1

273

( $\boldsymbol{U R S}$ urorectal septum, $\boldsymbol{U} \boldsymbol{G S}$ urogenital sinus, $\boldsymbol{C M}$ cloacal membrane, $\boldsymbol{H}$ hindgut).

274

275

276

277

278

279

week 6.

week 6.

Red rectangles in $\mathbf{a}, \mathbf{d}, \mathbf{g}$ and $\mathbf{j}$ are shown at higher magnification in $\mathbf{b}, \mathbf{e}, \mathbf{h}$ and $\mathbf{k}$, respectively. Green

rectangles in $\mathbf{b}, \mathbf{e}, \mathbf{h}$ and $\mathbf{k}$ are shown at higher magnification in $\mathbf{c}, \mathbf{f}, \mathbf{i}$ and $\mathbf{l}$, respectively.

Original magnification $100 \times(\mathbf{a}, \mathbf{d}, \mathbf{g}$ and $\mathbf{j}), 200 \times(\mathbf{b}, \mathbf{e}, \mathbf{h}$ and $\mathbf{k})$ and $400 \times(\mathbf{c}, \mathbf{f}, \mathbf{i}$ and $\mathbf{l})$.

\section{Figure 3. Spatiotemporal distribution of caudal-type homeobox proteins during gestation}


283 During gestation weeks 6, the cloacal membrane (CM) was very thin and elongated. CDX1-, CDX2- and

284 CDX4-positive cells were detected mainly in the mesenchyme of the urorectal septum (URS) and hindgut

285 (H). The cloacal membrane (CM) and urogenital sinus (UGS) were negative for CDX1, CDX2 and CDX4.

286 CDX2 and CDX4 immunoreactivity levels were lower compared to CDX1.

287 (URS urorectal septum, $\boldsymbol{U} \boldsymbol{G S}$ urogenital sinus, $\boldsymbol{C M}$ cloacal membrane, $\boldsymbol{H}$ hindgut).

288 Yellow rectangles in $\mathbf{a}, \mathbf{e}, \mathbf{i}$ and $\mathbf{m}$ are shown at higher magnification in $\mathbf{b}, \mathbf{f}, \mathbf{j}$ and $\mathbf{n}$, respectively.Red

289 rectangles in $\mathbf{b}, \mathbf{f}, \mathbf{j}$ and $\mathbf{n}$ are shown at higher magnification in $\mathbf{c}, \mathbf{g}, \mathbf{k}$ and $\mathbf{o}$, respectively. Green rectangles in

$290 \mathbf{c}, \mathbf{g}, \mathbf{k}$ and $\mathbf{o}$ are shown at higher magnification in $\mathbf{d}, \mathbf{h}, \mathbf{I}$ and $\mathbf{p}$, respectively.

291 Original magnification $40 \times(\mathbf{a}, \mathbf{e}, \mathbf{i}$ and $\mathbf{m}), 100 \times(\mathbf{b}, \mathbf{f}, \mathbf{j}$ and $\mathbf{n}), 200 \times(\mathbf{c}, \mathbf{g}, \mathbf{k}$ and $\mathbf{o})$ and $400 \times(\mathbf{d}, \mathbf{h}, \mathbf{l}$ and $\mathbf{~ p})$.

Figure 4. Spatiotemporal distribution of caudal-type homeobox proteins during gestation

\section{4 week 7.}

During gestation weeks 7, CDX1-, CDX2- and CDX4-positive cells were detected mainly in the mesenchyme

of the urorectal septum (URS) and hindgut (H). The cloacal membrane (CM) and urogenital sinus (UGS) were

CDX1.

(URS urorectal septum, $\boldsymbol{U} \boldsymbol{G S}$ urogenital sinus, $\boldsymbol{C M}$ cloacal membrane, $\boldsymbol{H}$ hindgut).

Yellow rectangles in $\mathbf{a}, \mathbf{e}, \mathbf{i}$ and $\mathbf{m}$ are shown at higher magnification in $\mathbf{b}, \mathbf{f}, \mathbf{j}$ and $\mathbf{n}$, respectively.Red

$\mathbf{c}, \mathbf{g}, \mathbf{k}$ and $\mathbf{o}$ are shown at higher magnification in $\mathbf{d}, \mathbf{h}, \mathbf{I}$ and $\mathbf{p}$, respectively. 
week 8.

During gestation weeks 8 , the rectum $(\mathrm{R})$ became separated completely from the urogenital sinus (UGS) and CDX1-, CDX2- and CDX4-positive cells disappeared from the mesenchyme. At the same time, the anorectal epithelium was positive for CDX1 and CDX4, and CDX1 immunoreactivity decreased gradually from the proximal rectum (R) to the anus. The anal epithelium was positive for CDX2.

317 ( $R$ rectum).

318 Yellow rectangles in $\mathbf{a}, \mathbf{d}, \mathbf{g}$ and $\mathbf{j}$ are shown at higher magnification in $\mathbf{b}, \mathbf{e}, \mathbf{h}$ and $\mathbf{k}$, respectively. Red

319 rectangles in $\mathbf{b}, \mathbf{e}, \mathbf{h}$ and $\mathbf{k}$ are shown at higher magnification in $\mathbf{c}, \mathbf{f}, \mathbf{i}$ and $\mathbf{l}$, respectively.

320 Original magnification $40 \times(\mathbf{a}, \mathbf{d}, \mathbf{g}$ and $\mathbf{j}), 100 \times(\mathbf{b}, \mathbf{e}, \mathbf{h}$ and $\mathbf{k})$ and $200 \times(\mathbf{c}, \mathbf{f}, \mathbf{i}$ and $\mathbf{l})$.

Figure 6. Spatiotemporal distribution of caudal-type homeobox proteins during gestation

\section{week 9.}

327 During gestation weeks 9, the anorectal epithelium was positive for CDX1 and CDX4, and CDX1

328 immunoreactivity decreased gradually from the proximal rectum $(\mathrm{R})$ to the anus. The anal epithelium was 
329 positive for CDX2.

330 ( $R$ rectum).

331 Yellow rectangles in $\mathbf{a}, \mathbf{d}, \mathbf{g}$ and $\mathbf{j}$ are shown at higher magnification in $\mathbf{b}, \mathbf{e}, \mathbf{h}$ and $\mathbf{k}$, respectively. Red

332 rectangles in $\mathbf{b}, \mathbf{e}, \mathbf{h}$ and $\mathbf{k}$ are shown at higher magnification in $\mathbf{c}, \mathbf{f}, \mathbf{i}$ and $\mathbf{l}$, respectively.

333 Original magnification $40 \times(\mathbf{a}, \mathbf{d}, \mathbf{g}$ and $\mathbf{j}), 100 \times(\mathbf{b}, \mathbf{e}, \mathbf{h}$ and $\mathbf{k})$ and $200 \times(\mathbf{c}, \mathbf{f}, \mathbf{i}$ and $\mathbf{l})$.

335 Figure 7. A summary schematic of Cdx proteins distribution pattern in rat and human embryo.

The distribution pattern of CDX proteins in humans differ markedly from those of the equivalent proteins in

rats. In rats, $\mathrm{Cdx} 1-, \mathrm{Cdx} 2-$ and $\mathrm{Cdx} 4$-positive cells were located in the cloacal/hindgut epithelium, whereas in

gestation weeks 4-7 and located in anal/anorectal epithelium during gestation weeks 8-9.

343 ( $\boldsymbol{U} \boldsymbol{R} \boldsymbol{S}$ urorectal septum, $\boldsymbol{U}$ urogenital sinus, $\boldsymbol{C M}$ cloacal membrane, $\boldsymbol{C} \boldsymbol{L}$ cloaca, $\boldsymbol{H}$ hindgut, $\boldsymbol{R}$ rectum).

344 (Red dots indicate distribution of $\mathrm{Cdx} 1$ protein. Yellow dots indicate distribution of $\mathrm{Cdx} 2$ protein. Green dots

345 indicate distribution of $\mathrm{Cdx} 4$ protein.) 